\title{
Miniature In Vitro Dissolution Testing of Powders for Inhalation
}

\author{
Sabine May ${ }^{1,2}$, Saskia Kind², Birte Jensen², Markus Wolkenhauer², \\ Marc Schneider ${ }^{1,3,4, *}$, and Claus-Michael Lehr ${ }^{1,4,5}$ \\ e-mail: Marc.Schneider@mx.uni-saarland.de \\ ${ }_{1}^{1}$ PharmBioTec GmbH, Saarbrücken, Germany \\ ${ }^{2}$ Boehringer Ingelheim Pharma GmbH \& Co KG, Ingelheim, Germany \\ ${ }^{3}$ Pharmaceutics and Biopharmacy, Philipps-Universität Marburg, Marburg, Germany \\ ${ }^{4}$ Biopharmaceutics and Pharmaceutical Technology, Saarland University, Saarbrücken, Germany \\ ${ }^{5}$ Helmholtz Institute for Pharmaceutical Sciences Saarland, Helmholtz Center for Infection Research, Saarbrücken, Germany
}

\section{ABSTRACT}

The aim of this study was the development and evaluation of a miniaturized setup for in vitro dissolution testing of inhalation powders based on an adapted Transwell system with artificial membranes. Such a system should be able to discriminate between different inhalation powders with good reproducibility by allowing easy handling while only small amounts of test compound and liquids are necessary.

The modified Transwell setup was tested by depositing a fraction of aerosol particles with an aerodynamic particle size less than $5 \mu \mathrm{m}$ on different artificial membranes (inserts) using a modified abbreviated Andersen cascade impactor to guarantee homogeneous and non-agglomerated particle distribution, which is essential for dissolution testing of powders for inhalation. The performance of the system was tested with the commercially available Transwell inserts and artificial membranes in a modified setup. Both systems provided reliable and comparable data that extends the flexibility of the test system with respect to the commercial setup. The dissolution tests were performed using budesonide and a proprietary active pharmaceutical ingredient (API), substance A, from the Boehringer Ingelheim research line. Besides the use of different membranes, the setup was modified to allow stirring, which improved the reproducibility of the dissolution process. Surprisingly, providing additional dissolution medium in the donor compartment for the poorly soluble substances decreased the stability of the dissolution process. Furthermore, the membrane material had a strong influence on the dissolution profile. The polyester (PE) and polycarbonate (PC) membranes interacted with the substances used, whereas regenerated cellulose and Isopore PC membranes were more suitable for the dissolution testing of these APIs.

KEYWORDS: Dissolution; Transwell; powders for inhalation.

\section{INTRODUCTION}

$\mathrm{N}$ ew pharmaceutical actives in development tend to be poorly soluble substances (1). Regarding in vitro tests for inhaled formulations, the focus has been primarily on their aerodynamic properties, aiming for maximizing pulmonary deposition $(2,3)$. However, the appearance of more and more poorly soluble actives for administration by inhalation necessitates that in vitro tests account for the special obstacles in the lungs such as deposition pattern and the low fluid amount. The large surface area of more than $100 \mathrm{~m}^{2}$ for deposition is counterbalanced by the small amount of aqueous fluid $\left(10-20 \mathrm{~mL} / 100 \mathrm{~m}^{2}\right)$ available for dissolution $(4,5)$. The successful integration of these airway specifics in an in vitro model for the drug dissolution of inhalation powders will be an intriguing and meaningful tool in addition to the study of aerodynamic properties (6).

\footnotetext{
*Corresponding author.
}

Several dissolution techniques for powders for inhalation have been described, such as the flow-through cell (7-9), the Franz diffusion cell (8-10), and the paddle apparatus equipped with a special sample holder $(8,11,12)$. The common disadvantage of these apparatus is the nonphysiologically large amount of dissolution medium (60-1000 mL). To overcome this limitation, Arora et al. (13) and Sakagami et al. (14) introduced a new approach based on Transwell inserts with a membrane providing an air interface and only a small amount of dissolution medium that can also be extended to a cell culturebased setup described by Haghi et al. $(10,15)$. In all these studies, general feasibility was the focus rather than critical factors such as controlling particle deposition, the influence of the membrane material, or the influence of stirring of the dissolution medium. The latter was addressed by Bhagwat et al. (16) but without any focus on the other factors. 
The aim of the present study was to establish and evaluate an adapted Transwell system for dissolution testing. Therefore, the deposition of particles with an aerodynamic diameter less than $5 \mu \mathrm{m}$ was carefully controlled. This deposition process allowed an exact quantification of the amount on the membrane, which is crucial for the dissolution process $(8,11)$. In consequence, we could specifically test several variables to identify critical factors, especially membrane material, addition of dissolution medium on the membrane (air side), and stirring of the acceptor medium, respectively. Overall, we were aiming to identify the most discriminating, robust, and reliable method for the dissolution testing of aerosol powders at an air interface based on a Transwell system.

\section{MATERIAL AND METHODS \\ Materials}

Different modifications (i.e., dibromide, crystalline, and amorphous base) of a research compound ("substance $A^{\prime \prime}$ ) were obtained from Boehringer Ingelheim (Ingelheim, Germany). Substance $A$ base and dibromide were micronized via jet milling. The amorphous base of substance A was produced by spray drying the crystalline base. Asa reference, the well-knownactive pharmaceutical ingredient (API) budesonide was purchased from Cipla (India). The crystallinity of budesonide was proved by DSC (data known shown). Solubility data for these substances are summarized in Table 1 as previously determined (8).

Table 1. Solubility Data of Substances in PBS Buffer pH 7.4 at $22{ }^{\circ} \mathrm{C}$

\begin{tabular}{|l|l|c|}
\hline \multicolumn{2}{|c|}{ Substance } & Solubility $(\mu \mathrm{g} / \mathrm{mL})$ \\
\hline Budesonide & & 17 \\
\hline \multirow{3}{*}{ Substance A } & amorphous base & 211 \\
\cline { 2 - 3 } & crystalline base & 7 \\
\cline { 2 - 3 } & dibromide & 265 \\
\hline
\end{tabular}

Transwell plates with polycarbonate (PC) membranes or polyester (PE) membranes (24-mm diameter and hence an area of $452.4 \mathrm{~mm}^{2}$ ) both with pore diameters of $0.4 \mu \mathrm{m}$ were obtained from Corning Costar (Corning, NY, USA). Regenerated cellulose membrane filters (100$\mathrm{mm}$ diameter, $0.45-\mu \mathrm{m}$ pore size) were purchased from Whatman (Dassel, Germany). Isopore membrane filters consisting of PC (80-mm diameter) with a 0.4- $\mu \mathrm{m}$ pore size were obtained from Millipore (Cork, Ireland). Isopore and regenerated cellulose membranes were manually cut to the Transwell insert membrane size (25 $\mathrm{mm})$. The membrane pore size was chosen to allow the dissolved molecules to pass through the membrane but prevent undissolved substance particles from crossing the membrane. The powder had a large amount of small particles, and even $10 \%$ of the particles had a diameter of about $0.5 \mu \mathrm{m}$ (data not shown). Therefore, membranes with pore diameters of 0.4 and $0.45 \mu \mathrm{m}$ were chosen.

Phosphate buffered saline (PBS) with a pH of 7.4 was used as the dissolution medium for all experiments.

The HPLC eluent was $\mathrm{pH} 3 \mathrm{KH}_{2} \mathrm{PO}_{4}$ buffer (Merck, Darmstadt, Germany). Acetonitrile (HPLC grade) and PBS buffer tablets were purchased from Sigma Aldrich Chemicals (Steinheim, Germany). HPLC vials were obtained from Macherey Nagel (Düren, Germany) and Waters (Milford, USA); vial inserts were from Wicom (Heppenheim, Germany). A Milli-Q System (Millipore, Molsheim, France) was used to obtain purified water, which was used for all experiments.

\section{Methods}

Scanning Electron Microscopy

The different membrane types were mounted on commercial sample holders and imaged by scanning electron microscopy. The membrane samples were sputter-coated with approximately $5 \mathrm{~nm}$ platinum with a Precision Etching Coating System (Model 682, Gatan, München, Germany). The microscope (Leo, Gemini Supra 55 VP with SE detector) was operated at an acceleration voltage of $1 \mathrm{kV}$ for imaging.

\section{High Performance Liquid Chromatography (HPLC)}

Quantification of budesonide and substance $A$ was performed by HPLC (Alliance system, Waters $\mathrm{GmbH}$, Frankfurt, Germany) using a reversed-phase column (LiChrosphor 60 RP Select B, $60 \times 4$ mm; MZ Analysentechnik, Mainz, Germany) and a UV-vis detector at $\lambda_{\mathrm{Abs}}=240 \mathrm{~nm}$ for budesonide and $225 \mathrm{~nm}$ for substance A. The mobile phase was composed of $\mathrm{pH} 3$ buffer/ acetonitrile $(60 / 40$ for budesonide, $65 / 35$ for substance A). The eluent flow rate was set to $1.7 \mathrm{~mL} / \mathrm{min}$. The column temperature was $40^{\circ} \mathrm{C}$, and the injection volume of each sample was $10 \mu \mathrm{L}$.

\section{Contact Angle Measurement}

For the determination of membrane surface polarity, contact angle measurements were performed with a Drop Shape Analysis System (DSA 10 MK 2 Krüss, Germany). For the measurement, the advancing sessile drop method was used with purified water at room temperature $\left(23^{\circ} \mathrm{C}\right)$. A drop was formed (flow rate of $16 \mu \mathrm{L} / \mathrm{min}$ ) and the final volume of $8 \mu \mathrm{L}$ was observed. Images of the illuminated droplet were taken by a camera mounted vertically to the sample table, and the contact angle was determined. For each membrane material, five drops were analyzed. During droplet growth, the 
software calculated the advancing contact angle every second for $15 \mathrm{sec}$. The resulting value was the average of all measurements made within the 15 -sec interval.

\section{Particle Collection on Membrane Filters}

For dose collection, an abbreviated Andersen cascade impactor (ACl) with a stage extension (SE) inserted between the filter stage and stage one (Figure 1) was used, as described previously (11). Substances were filled into polyethylene capsules and dispersed with a HandiHaler (Boehringer Ingelheim, Germany) at standard USP conditions ( $4 \mathrm{kPa}, 4 \mathrm{~L}$ ). Suction time was shortened to $0.85 \mathrm{sec}$ to allow sedimentation of particles on the membrane during a lag time of $5 \mathrm{~min}$.

For a better comparison of the different substances,

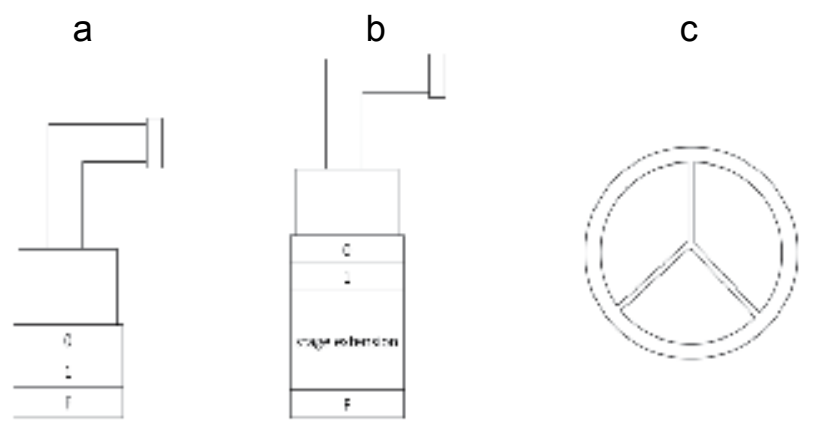

Figure 1. (a) Abbreviated $A C l$, (b) abbreviated $A C l$ with stage extension, and (c) modified filter stage.

it was necessary to deposit similar amounts of every substance on the membrane. Therefore, different substances required individual amounts weighed into the capsule ( $0.5 \mathrm{mg}$ up to $4 \mathrm{mg}$ ) to compensate for different dispersion behavior (11).

For dose collection, three different approaches were used. Test method 1 was used for commercially available Transwell inserts; approaches 2 and 3 used a modified Transwell for the different membrane types:

\section{(1) PE and PC Transwell membranes}

For dose collection, an abbreviated $\mathrm{ACl}$ with stage extension with normal filter stage was used. A cover with three voids for the Transwell insert was placed into the filter stage. The Transwell inserts were positioned onto the filter stage (and in the voids). The inner wall of each insert itself was covered with a polyetherketone (PEEK) cover avoiding particle deposition on the inner and outer wall of the insert (Figure 2). This cover is very thin and does not hinder sedimentation of particles on the Transwell insert membrane. After dose collection, the PEEK cover was removed. The PEEK cover was necessary to avoid particle deposition on the inner and outer wall, which cannot be quantified. The amount deposited on the PEEK cover can be determined and avoids an overestimation of the amount of substance deposited on the membrane.

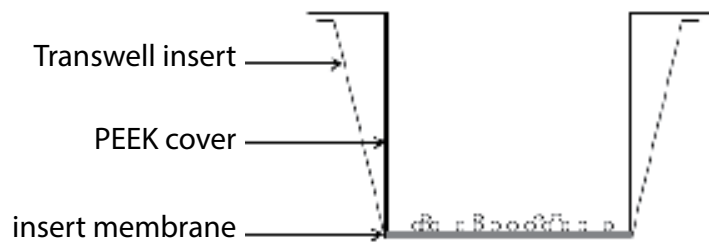

Figure 2. Transwell insert with removable PEEK cover to restrict drug deposition to the membrane and not to the insert walls.

(2) Regenerated cellulose membrane (RC) In addition to the stage extension, a modified filter stage was used to allow a homogeneous deposition (Figure 1c) (11). After particle deposition, the membrane was cut to the size of the Transwell insert (25-mm diameter). These membranes were then place with deposited particles facing up into an adapted Transwell insert providing a rim with a small sieve to position the membrane.

(3) Isopore PC membrane (IPC)

The IPC membrane has a very thin appearance and is not stable on the modified filter stage during dose collection. Hence, use of the normal filter stage was necessary. The procedure after dose collection was the same as for setup 2 .

\section{The Transwell System as Dissolution Apparatus}

Similar to the Franz Cell (17) used in skin drug delivery research, the modified Transwell offers an air-liquid interface, but with significantly smaller amounts of dissolution medium $(13,14)$.

Release profiles of aerodynamically classified particles of substance $A$ in its different modifications and budesonide were investigated using the adapted Transwell.

\section{Adapted Transwell}

To allow for stirring the commercially available Transwell system, consisting of the base plate with six wells (24-mm diameter), inserts, and a lid to cover the system, a spacer plate (2-mm thickness) was constructed to allow the inserts to be lifted to fit stir bars in the receptor chamber. To facilitate sampling from the receptor compartment, small holes for each well were drilled into the lid covering the setup. According to a defined time schedule, sampling was done manually with a syringe (100 Sterican needle: Braun, Melsungen, Germany; $1 \mathrm{~mL}$ disposable 
syringe: Wicom, Heppenheim, Germany). The solvent removed during sampling $(0.2 \mathrm{~mL})$ was refilled with fresh prewarmed PBS buffer $\left(37^{\circ} \mathrm{C}\right)$ to maintain a constant volume (Figure 3a). At the end of the experiment, each insert was rinsed with $25 \mathrm{~mL}$ of solvent according to the substance used to determine the adsorbed or remaining drug amount on the membrane. Acetonitrile was used for budesonide, substance $A$ amorphous base, and substance A crystalline base; $\mathrm{pH} 7.4$ PBS buffer was used for substance A dibromide.

\section{Modified Transwell}

For investigating different membrane materials (regenerated cellulose and the Isopore membrane), the Transwell insert was modified. The membrane was cut out, and a small plastic edge was thermoformed. A small metal sieve was placed on this edge as support for the membranes. The modified inserts were reusable in contrast to the standard Transwell inserts (Figure 3b).
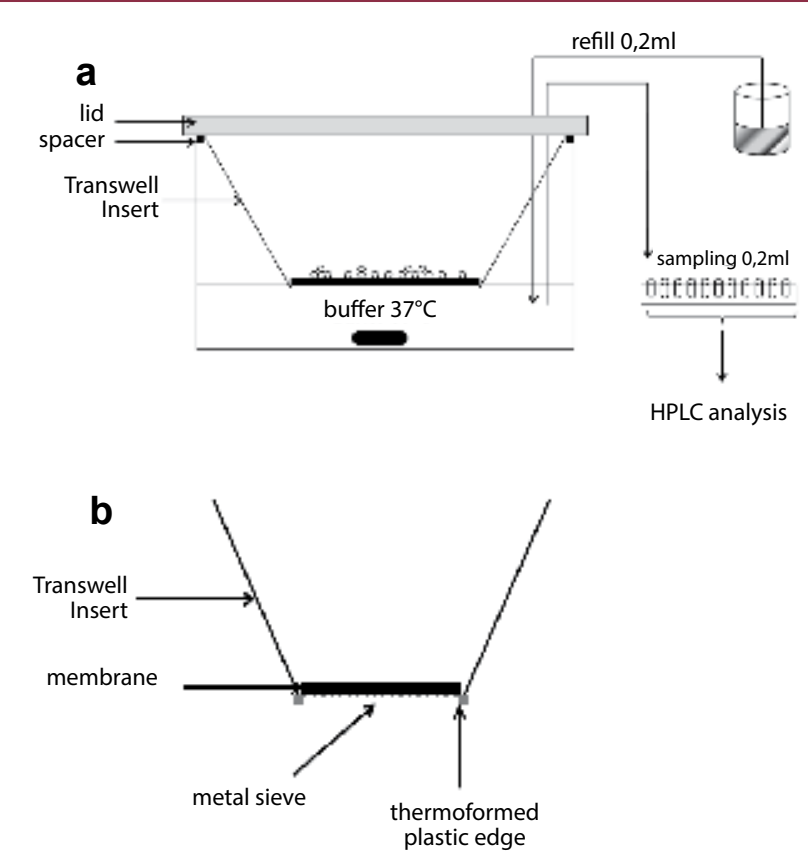

Figure 3. Schematic drawings of (a) adapted Transwell dissolution apparatus and (b) modified Transwell insert.

An aliquot of dissolution medium $(2.6 \mathrm{~mL}$ for the commercially available inserts and $3.85 \mathrm{~mL}$ for the adapted inserts) was placed into the acceptor compartment. The membranes with particles facing up were touching the dissolution medium, ensuring an air-liquid interface with no hydrostatic pressure on the system (Figure 3a).

Dissolution tests were performed at $37{ }^{\circ} \mathrm{C}$ and $100 \% \mathrm{RH}$ in a climate cabinet (Espec climate cabinet, Weilburg, Germany) using degassed PBS buffer $\mathrm{pH} 7.4$ as dissolution medium. The high humidity was necessary to avoid evaporation of the dissolution medium. Sink conditions ( $10 \%$ of saturation solubility) were ensured during the experiments. This was realized by the conditions of the sampling process $(0.2 \mathrm{~mL}$ of $2.5 \mathrm{~mL}$, equal to $8 \%$ of the dissolution medium, and $5 \%$ of $3.85 \mathrm{~mL}$ ) and the chosen fine particle dose (FPD) on the membrane

\section{Drug Quantification}

Drug concentrations in the acceptor compartment were determined by HPLC. The total amount of drug initially loaded on the membranes was calculated using the maximum of the cumulatively released amounts plus the remaining particles on the membrane (determined at the end of each experiment). The amount of drug released was calculated with eq 1 where $m$ is the amount of drug released, $V_{\text {medium }}$ is the volume of dissolution medium (2.6 or $3.85 \mathrm{~mL})$, and $V_{\text {sampling }}$ is the sampled volume $(0.2$ $\mathrm{mL})$.

$$
m_{t=1}=C_{t=1} \times V_{\text {medium }}-\left[C_{t=i-1} \times\left(V_{\text {medium }}-V_{\text {sampling }}\right)\right]+m_{t=i-1}
$$

\section{Membrane Permeation Test}

To investigate the influence of the membrane material on the dissolution behavior, the diffusion of the drug across the membrane was studied. Comparing the diffusion behaviors of the compounds across different membrane materials and types allowed identification of interactions between the compound and the membrane. To ensure the same conditions for all membrane types, these tests were performed with a small Franz cell-like setup instead of the Transwell system. The acceptor compartment was filled with $7.25 \mathrm{~mL}$ PBS buffer. The test membrane was clamped between the acceptor chamber and the upper cylinder of the cell, and the acceptor chamber was fixed with an external spring on the receptor chamber. The dissolution medium in the acceptor chamber was stirred at $140 \mathrm{rpm}$. To determine the diffusion of dissolved substance through the membrane, $100 \mu \mathrm{L}$ of API solution at a concentration of $10 \mu \mathrm{g} / \mathrm{mL}$ was placed on the membrane. Manual sampling of $200 \mu \mathrm{L}$, which was then replaced by the same volume of PBS buffer, was performed through the small side arm at the same time points as for the Transwell system. The membrane permeation test was performed in triplicate at $37^{\circ} \mathrm{C}$ and $100 \% \mathrm{RH}$ in a climate cabinet.

\section{Stirring}

The influence of a stirred versus a non-stirred acceptor on the dissolution process was determined for $140 \mathrm{rpm}$. Stirring should guarantee a homogeneous concentration and should reduce concentration-based diffusion effects. After dose collection, the inserts were placed into the 
adapted Transwell system. The dissolution tests were performed without additional dissolution medium on the membrane because the results indicated better performance without additional dissolution medium (see Results section).

\section{Effect of Powder Dissolution and Diffusion by Addition of Dissolution Medium on the Membrane}

Adding additional dissolution medium on the membrane as used by Arora et al. (13) is thought to facilitate drugfluid contact and improve drug dissolution and hence diffusion across the membrane.

After dose collection, the inserts were placed into the adapted Transwell dissolution system. Immediately after placing the insert, $40 \mu \mathrm{L}$ of PBS buffer was added onto each membrane, and the lid was closed. The tests were performed with and without this additional $40 \mu \mathrm{L}$ and with stirring (140 rpm).

\section{Comparison of Different PC Membranes}

Because different results were obtained with the two different polycarbonate membranes (PC and IPC), two additional tests with budesonide and the two types of PC membranes were performed. The test with the modified insert should show if the difference between the two membrane types is due to material or test setup. As the modified Tranwell accommodates the membrane on a grid, the open edges might also allow for diffusion. To address this for the commercial, closed system, the edge of the original PC Transwell membrane insert was perforated with a small needle (0.5-mm diameter). Easier access of dissolution medium between the upper side of membrane and acceptor medium should be possible with the perforation. This procedure should allow a comparison of the dissolution effect to the membrane in the modified Transwell. The second approach was based on cutting the membrane with deposited drug out of the commercial Transwell and placing it into the modified Transwell insert (as described above for RC and IPC). The two tests were performed in triplicate for budesonide with stirring and without additional dissolution medium on the membrane. These two approaches should allow us to investigate the effect of the Transwell modification on the dissolution and to compare the self-made to the commercial setup.

Table 2 summarizes the experiments for testing the influence of different membrane materials, stirring, and adding additional dissolution medium.

\section{Data Treatment}

From the experiments, a mean dissolution curve with standard deviations was calculated and used for further analysis.
Table 2. Overview of Experimental Procedures

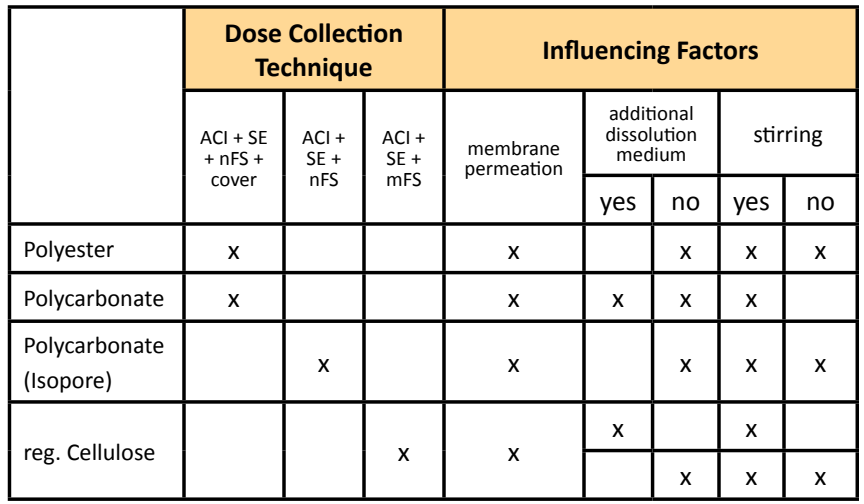

Modified filter stage: $m F S$; normal filter stage: $n F S$; stage extension: $S E$. Experiments performed are marked with an $x$. All experiments were done in triplicate.

Comparison of the different setups and substances was based mainly on the mean dissolution time $(M D T) \pm$ standard deviation. MDT was calculated with eq 2 (18):

$$
M D T=\frac{\sum_{i=t}^{n} \bar{t}_{l} \Delta M_{i}}{\sum_{i}^{n} \Delta M_{i}}
$$

where $t_{\mathrm{i}}$ is the middle of the respective time intervals between sampling points and $\Delta M_{i}$ is the amount of API dissolved in these intervals.

Additionally, dissolution profiles were compared using difference and similarity factors $\left(f_{1}\right.$ and $\left.f_{2}\right)$. The difference (eq 3 ) and the similarity (eq 4) factors are both modelindependent approaches, directly comparing the difference between percent drug released per unit time for a test and reference product $(19,20)$ :

$$
\begin{gathered}
f_{1}=\left\{\left[\sum_{t=1}^{n}\left|R_{t}-T_{t}\right|\right] /\left[\sum_{t=1}^{n} R_{t}\right]\right\} \times 100 \\
f_{2}=50 \cdot \log \left\{\left[1+\frac{1}{n} \sum_{t=1}^{n}\left(R_{t}-T_{t}\right)^{2}\right]^{-0.5} \times 100\right\}
\end{gathered}
$$

where $n$ is the number of dissolution samples and $R_{\mathrm{t}}$ and $T_{\mathrm{t}}$ are the mean percentage drug released at each time point for reference and test product, respectively. For two curves to be considered similar, $f_{1}$ must be less than $15\left(f_{1}<15\right)$ and $f_{2}$ must be greater than $50\left(f_{2}>50\right)(19$, 20). 

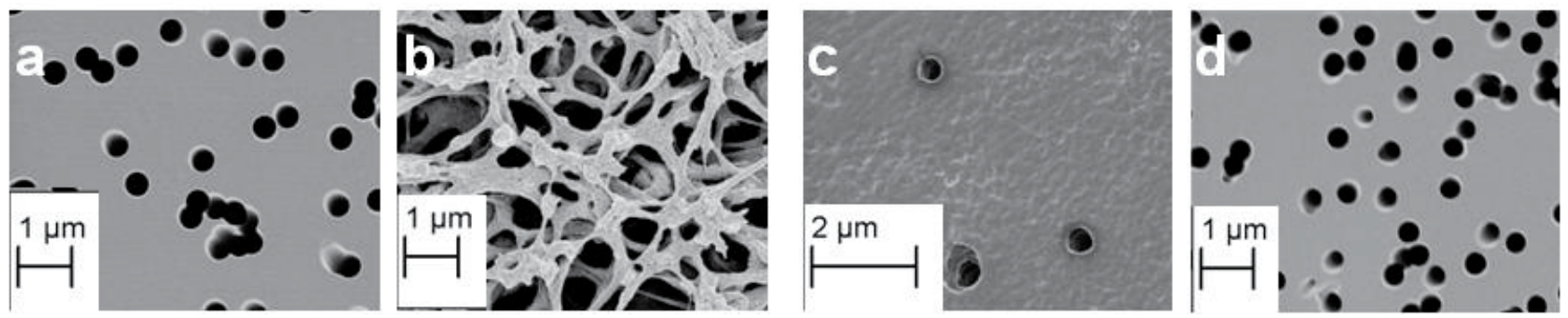

Figure 4. SEM images of (a) Isopore PC, (b) regenerated cellulose, (c) Transwell-PE, and (d) Transwell PC.

\section{RESULTS}

\section{Scanning Electron Microscopy}

SEM micrographs of the membranes (Figure 4) demonstrate the different structures of the membranes. PC and PE membranes are both tracked-etched filters, having more or less straight pores crossing the membrane. The commercially available PC Transwell membrane was very similar to the IPC membrane. In comparison, the regenerated cellulose membrane (RC) has a more sponge-like structure. Furthermore, SEM micrographs demonstrate the different pore numbers per square centimeter, especially for PE $\left(4 \times 10^{6}\right.$ pores $\left./ \mathrm{cm}^{2}\right)$ and PC $\left(1 \times 10^{8}\right.$ pores $\left./ \mathrm{cm}^{2}\right)$.

\section{Contact Angle Measurement}

Table 3 shows the results of the contact angle measurements. The regenerated cellulose shows complete wetting $\left(\theta<10^{\circ}\right)$. The contact angles for PC and PE membranes were less than $90^{\circ}$, hence the chosen membranes had a more hydrophilic surface. A comparison of Isopore and Transwell PC membranes showed that the PC membrane has a slightly poorer wettability than the PE membrane. In addition, the two Transwell membranes showed a lower reproducibility for the contact angle measurement than the Isopore membrane.

Table 3. Contact Angle for the Different Membranes

\begin{tabular}{|l|c|c|c|}
\hline & $\begin{array}{c}\text { Isopore } \\
\text { polycarbonate }\end{array}$ & $\begin{array}{c}\text { Transwell } \\
\text { polycarbonate }\end{array}$ & $\begin{array}{c}\text { Transwell } \\
\text { polyester }\end{array}$ \\
\hline water $\Theta\left({ }^{\circ}\right)$ & $57.4 \pm 1.9$ & $63.7 \pm 9.3$ & $43.5 \pm 4.2$ \\
\hline
\end{tabular}

Mean $\pm S D ; n=4 ; 15$ measurements per drop.

\section{Membrane Permeation Test}

The membrane permeation tests showed substancespecific differences for the diffusion of the different API solutions through the membrane. For substance A (Figure
$5 a, b, c)$, less than $80 \%$ of API solution diffused through the membranes; for budesonide, the diffused amount reached almost $90 \%$ within 60 min (Figure 5d). The PE membrane showed slower diffusion for all substances. The Isopore PC membrane and the regenerated cellulose membrane showed comparable amounts with fast diffusion. Depending on the substance and membrane material, the error bars are quite large due to the difficult sampling procedure and the small amounts of diffused substances.

\section{Stirring}

Figure 6 demonstrates that for budesonide and substance A crystalline base with the IPC and the RC membranes, stirring led to faster dissolution and higher dissolved amounts for the tested substances (dibromide and amorphous base, data not reported). As expected from the membrane permeation test using the PE membrane, the dissolution profiles show a lower permeated amount (less than 60\% dissolved). With the PE membrane, the amount of dissolved budesonide substance (30\%) in the unstirred setup was higher than that in the stirred setup (<20\%). This result is the opposite of those for the other membranes and substances (Figure 6). Overall, the dissolution process was usually faster for the stirred setup than for the unstirred setup for all tested membranes and substances.

The profiles of substance A crystalline base were similar for the stirred and the unstirred setup using PE and IPC membranes (Figure 6a,c). With the RC membrane, the dissolution process was faster when the dissolution medium was stirred (Figure 6b).

\section{Addition of Dissolution Medium on the Membrane}

The use of additional dissolution medium on the membrane (13) was expected to facilitate contact of the particle with the dissolution medium and hence increase 

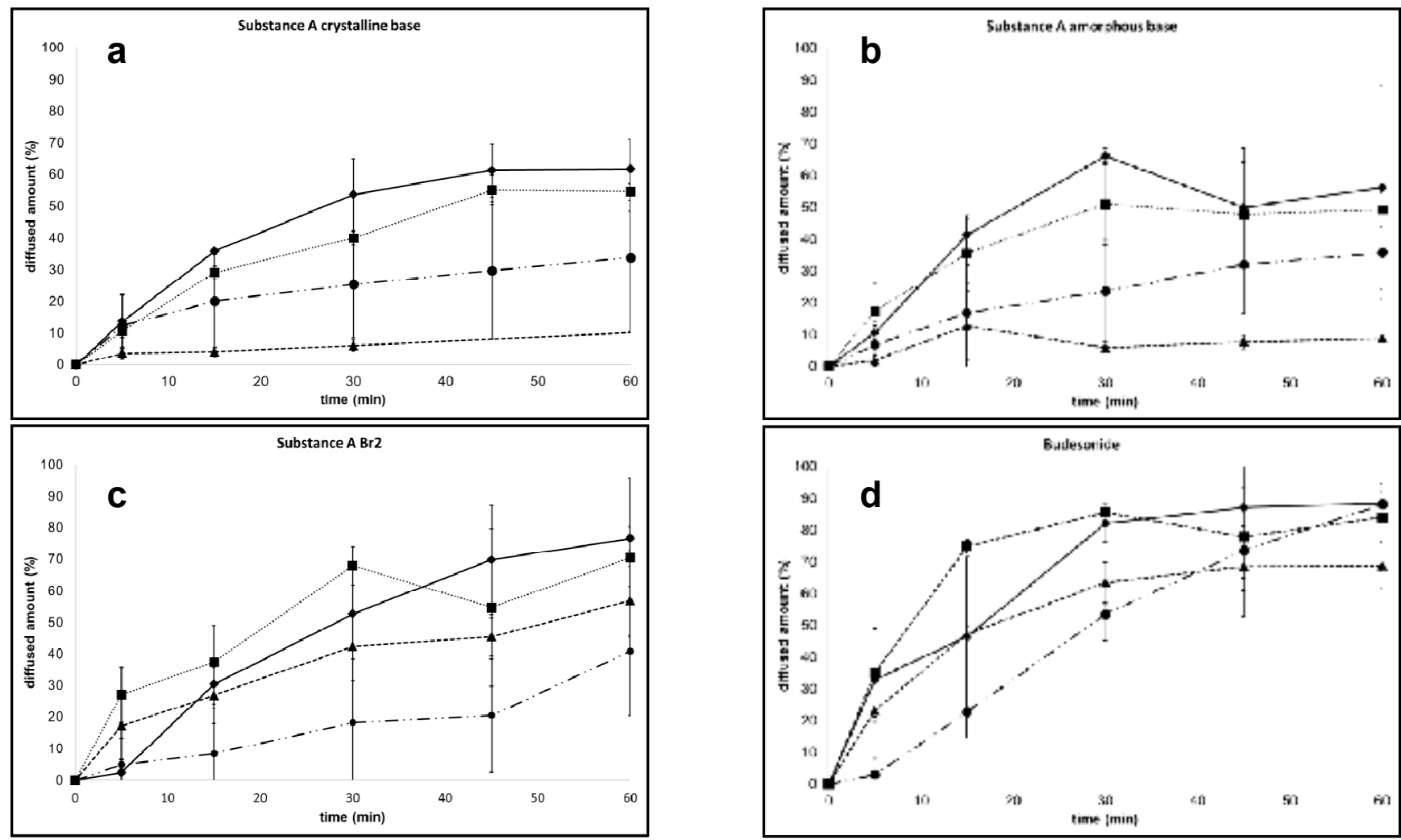

Figure 5. Membrane permeation test for $(\bullet)$ regenerated cellulose, $(\mathbf{\Delta}$, dashed line) Transwell $P C,(\boldsymbol{\square}$, dotted line) Isopore $P C$, and $(\bullet$, dashed-dotted line) $P E$ membrane with (a) substance A crystalline base, (b) amorphous base, (c) bromide, and (d) budesonide (mean $\pm S D, n=3$, SD). Error bars exist in all cases but are sometimes too small to be displayed.
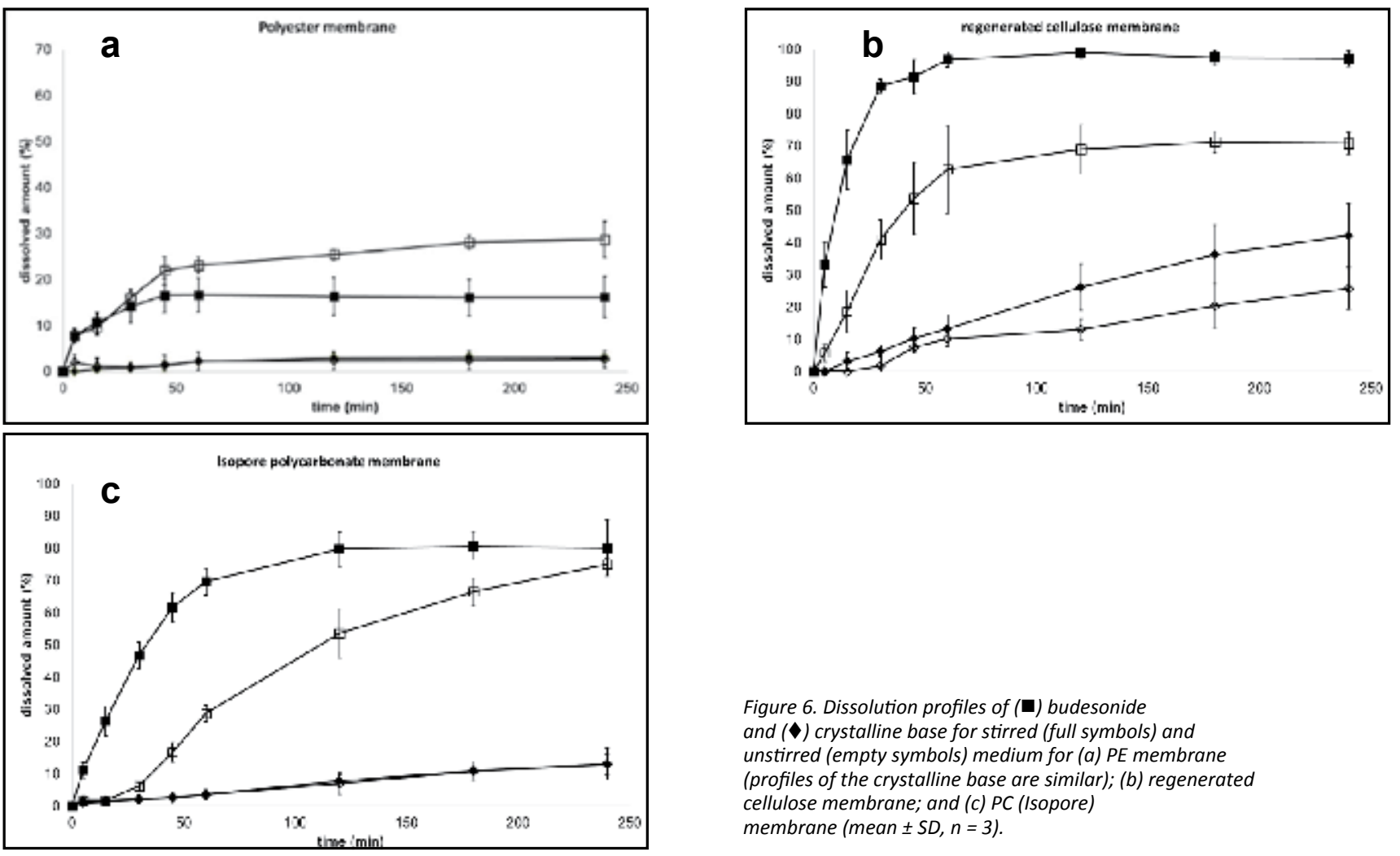

Figure 6. Dissolution profiles of (घ) budesonide and ( $)$ crystalline base for stirred (full symbols) and unstirred (empty symbols) medium for (a) PE membrane (profiles of the crystalline base are similar); (b) regenerated cellulose membrane; and (c) PC (Isopore) membrane (mean $\pm S D, n=3$ ). 
the reproducibility and speed of the dissolution process. A detailed view of the profiles for the different substances reveals substance-dependent differences (Figure 7). The profiles for budesonide using a regenerated cellulose membrane showed no difference in the first $20 \mathrm{~min}$. Finally, the dissolution profile without additional medium reached almost $100 \%$ with small error bars. In contrast, the profile with additional dissolution medium leveled off at an average of $70 \%$ with large error bars. The MDT for both profiles was also different (16.6 $\pm 0.7 \mathrm{~min}$ vs $12.8 \pm$ $2.6 \mathrm{~min}$, Table 4).

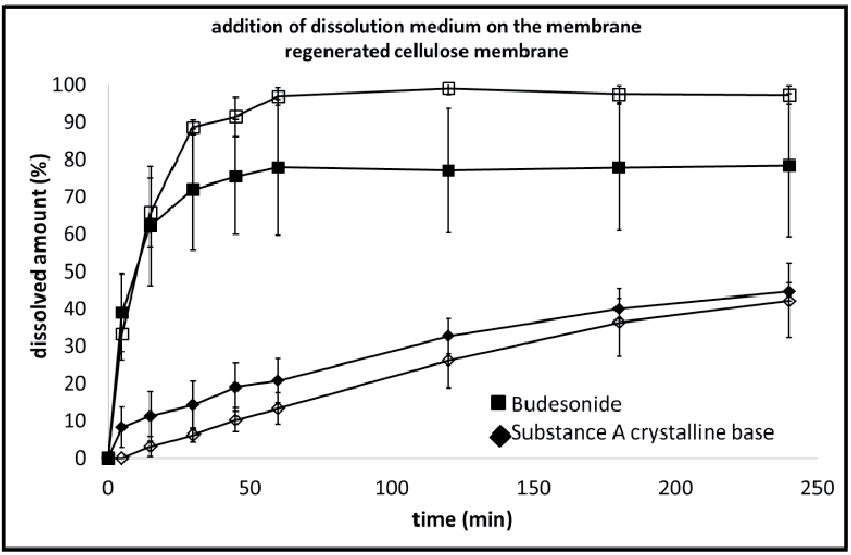

Figure 7. Influence of additional dissolution medium on the $R C$ membrane on the dissolution process of budesonide and substance A crystalline base. Full symbols with, open symbols without additional dissolution medium on the membrane (mean $\pm S D$, $n=3$, dissolution medium is stirred).

The beginning of the dissolution process for substance $A$ crystalline base with additional dissolution medium on the membrane was faster than the process without. In the progress of dissolution, the two profiles converged. As expected, due to the low solubility of substance A crystalline base, the dissolution process was quite slow. MDT calculation was not meaningful because in the observed time, the plateau of the dissolution profile was not reached.

For substance $A$ amorphous base and dibromide, the dissolution profiles (data not reported) with and without additional dissolution medium are similar. In contrast, the differences in calculated MDT values (Table 4) were not reflected in the dissolution profiles for substance $A$ dibromide (data not reported). The MDT for the dibromide with additional dissolution medium was $14.6 \pm 3.0$ min and $6.5 \pm 2.8 \mathrm{~min}$ without additional medium. Based on calculated $f_{1}$ and $f_{2}$ values, the dissolution profiles for substance $A$ amorphous base $\left(f_{1}=6.7 ; f_{2}=62.2\right)$ and dibromide $\left(f_{1}=11.5 ; f_{2}=55.2\right)$ were similar to each other. For budesonide, the tests indicate different profiles $\left(f_{1}=\right.$ $\left.21.6 ; f_{2}=40.4\right)$.
Table 4. Summary of Substances and Membrane Material With Corresponding Dissolution Setup

\begin{tabular}{|c|c|c|c|c|c|}
\hline \multirow[t]{2}{*}{ Substance } & \multirow[t]{2}{*}{$\begin{array}{c}\text { Membrane } \\
\text { material }\end{array}$} & \multirow{2}{*}{$\begin{array}{l}\text { diss } \\
\text { med } \\
\text { add }^{a}\end{array}$} & \multirow[t]{2}{*}{$\mathrm{rpm}^{b}$} & $\begin{array}{l}\text { recalculated FPD } \\
\text { on filter }(\mu \mathrm{gg})^{c}\end{array}$ & $M D T(\min )$ \\
\hline & & & & mean $\pm \mathrm{SD}$ & mean \pm SD \\
\hline \multirow{4}{*}{ Budesonide } & PC & + & $\begin{array}{l}+ \\
+\end{array}$ & $\begin{array}{l}5.5 \pm 0.7 \\
5.9 \pm 1.5\end{array}$ & n.p. ${ }^{d}$ \\
\hline & $\mathrm{RC}$ & $\begin{array}{l}+ \\
- \\
-\end{array}$ & $\begin{array}{l}+ \\
+ \\
-\end{array}$ & $\begin{array}{l}3.9 \pm 1.5 \\
4.2 \pm 0.2 \\
5.2 \pm 0.6\end{array}$ & $\begin{array}{c}12.8 \pm 2.6 \\
16.6 \pm 0.7 \\
40.6 \pm 13.3\end{array}$ \\
\hline & PE & - & + & $\begin{array}{l}4.4 \pm 0.5 \\
4.4 \pm 0.5\end{array}$ & $\begin{array}{c}21.6 \pm 8.9 \\
40.5 \pm 15.8\end{array}$ \\
\hline & IPC & - & + & $\begin{array}{l}15.2 \pm 1.0 \\
17.7 \pm 0.5\end{array}$ & $\begin{array}{l}40.4 \pm 6.3 \\
94.6 \pm 4.6\end{array}$ \\
\hline \multirow{4}{*}{$\begin{array}{c}\text { Substance } \\
\text { A crystalline } \\
\text { base }\end{array}$} & PC & $\begin{array}{l}+ \\
-\end{array}$ & $\begin{array}{l}+ \\
+\end{array}$ & $\begin{array}{l}5.6 \pm 1.7 \\
6.7 \pm 5.0\end{array}$ & n.p. \\
\hline & $\mathrm{RC}$ & $\begin{array}{l}+ \\
- \\
-\end{array}$ & $\begin{array}{l}+ \\
+ \\
-\end{array}$ & $\begin{array}{l}3.8 \pm 0.5 \\
3.8 \pm 0.6 \\
2.3 \pm 0.1\end{array}$ & n.p. \\
\hline & $\mathrm{PE}$ & $\begin{array}{l}- \\
-\end{array}$ & + & $\begin{array}{l}5.2 \pm 0.5 \\
3.5 \pm 0.7\end{array}$ & n.p. \\
\hline & IPC & $\begin{array}{l}- \\
-\end{array}$ & + & $\begin{array}{l}15.8 \pm 1.3 \\
16.9 \pm 1.5\end{array}$ & n.p. \\
\hline \multirow{4}{*}{$\begin{array}{c}\text { Substance } A \\
\text { amorphous } \\
\text { base }\end{array}$} & PC & $\begin{array}{l}+ \\
-\end{array}$ & $\begin{array}{l}+ \\
+\end{array}$ & $\begin{array}{l}26.2 \pm 5.4 \\
37.8 \pm 5.6\end{array}$ & n.p. \\
\hline & $\mathrm{RC}$ & $\begin{array}{l}+ \\
- \\
-\end{array}$ & $\begin{array}{l}+ \\
+ \\
-\end{array}$ & $\begin{array}{l}9.0 \pm 0.5 \\
8.6 \pm 0.9 \\
1.8 \pm 0.1\end{array}$ & $\begin{array}{c}6.9 \pm 1.5 \\
13.6 \pm 10.1 \\
29.7 \pm 6.9\end{array}$ \\
\hline & $\mathrm{PE}$ & $\begin{array}{l}- \\
-\end{array}$ & + & $\begin{array}{c}6.5 \pm 1.0 \\
10.4 \pm 3.3\end{array}$ & n.p. \\
\hline & IPC & $\begin{array}{l}- \\
-\end{array}$ & + & $\begin{array}{l}17.6 \pm 4.3 \\
33.1 \pm 7.6\end{array}$ & $\begin{array}{l}16.9 \pm 3.9 \\
27.9 \pm 4.7\end{array}$ \\
\hline \multirow{4}{*}{$\begin{array}{c}\text { Substance A } \\
\text { dibromide }\end{array}$} & PC & $\begin{array}{l}+ \\
-\end{array}$ & $\begin{array}{l}+ \\
+\end{array}$ & $\begin{array}{c}7.1 \pm 1.5 \\
12.0 \pm 1.2\end{array}$ & n.p. \\
\hline & $\mathrm{RC}$ & $\begin{array}{l}+ \\
- \\
-\end{array}$ & $\begin{array}{l}+ \\
+ \\
-\end{array}$ & $\begin{array}{l}2.2 \pm 0.2 \\
3.2 \pm 0.5 \\
1.6 \pm 1.0\end{array}$ & $\begin{array}{c}14.6 \pm 3.0 \\
6.5 \pm 2.8 \\
36.4 \pm 9.7\end{array}$ \\
\hline & PE & $\begin{array}{l}- \\
-\end{array}$ & + & $\begin{array}{l}3.5 \pm 1.6 \\
1.6 \pm 0.5\end{array}$ & n.p. \\
\hline & IPC & $\begin{array}{l}- \\
-\end{array}$ & + & $\begin{array}{c}19.6 \pm 2.5 \\
4.9 \pm 0.3\end{array}$ & $\begin{array}{c}4.4 \pm 0.5 \\
24.7 \pm 7.0\end{array}$ \\
\hline
\end{tabular}

${ }^{a}$ diss med add: additional dissolution medium on the membrane

${ }^{b}$ with (+) or without (-) stirring at $100 \mathrm{rpm}$

$c$ recalculated FPD on filter (mean $\pm S D$ ) and MDT (mean $\pm S D), n=3$

${ }^{d}$ n.p.: calculation not possible

The dissolution profiles with additional dissolution medium on the membrane showed reproducibility similar to or lower than those without additional dissolution medium, with the exception of the amorphous base (data not reported).

The dissolution profiles using the PC membrane with additional dissolution medium on the membrane were similar to those for RC (data not reported). However, dissolution profiles of all substances did not exceed $20 \%$ of drug in the receptor compartment (budesonide and 
substance A crystalline base shown in Figure 8), which is unexpected in view of the membrane permeation test results (Figure 5).

Figure 8 demonstrates the differences between the two PC membranes for budesonide and substance A crystalline base. The dissolution profile of budesonide using the IPC membrane reached $80 \%$ of the dissolved amount; the profile of the PC membrane only $10 \%$. The profiles of substance A crystalline base are similar in the first 60 min. Later the dissolution profile using IPC membrane increased, whereas the profile using PC membrane remained at less than $10 \%$ of the dissolved amount. The results for the amorphous base and dibromide (data not reported; MDT in Table 4) reveal similar behavior. With the IPC membrane, substance A dibromide reached $90 \%$ dissolved within $10 \mathrm{~min}$ and substance $A$ amorphous base reached $70 \%$. In contrast, the profiles using the PC membrane showed less than $15 \%$ dissolved API. It is remarkable that the reproducibility of the dissolution profiles for both PC and IPC was excellent.

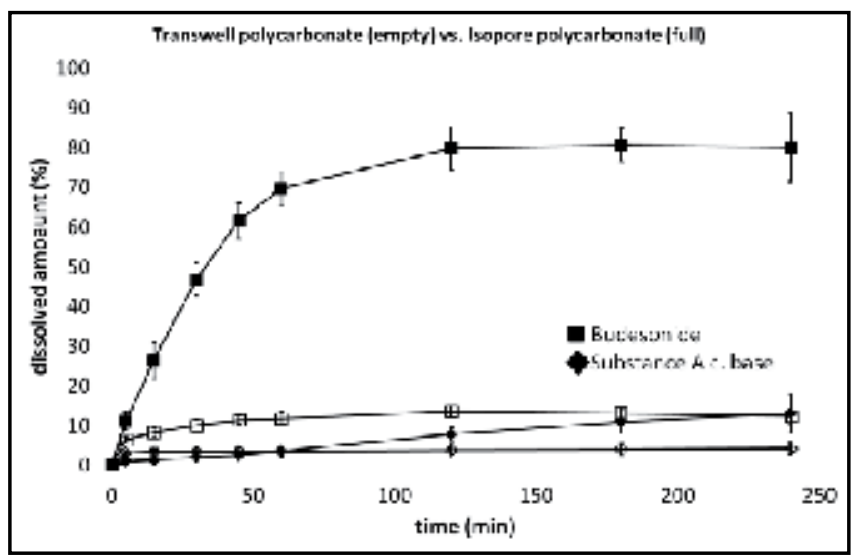

Figure 8. Dissolution profiles of budesonide and substance $A$ crystalline base with the use of PC and IPC membranes. Setup was with stirring of the dissolution medium and without

additional dissolution medium on the membrane (mean $\pm S D$, $n=3)$. Error bars exist in all cases but are too small to be displayed.

\section{Comparison of the Different PC Membranes}

As described in the method section, different results were obtained for the two different PC (i.e., PC and IPC) membranes (Figure 8). Hence, two additional tests with budesonide and the two types of PC membranes were performed.

Surprisingly, as shown in Figure 9 perforating the edge of the membrane with a needle or cutting the PC membrane out of the Transwell and placing into the adapted Transwell did not result in a faster dissolution and diffusion of budesonide through the membrane.
The dissolution profiles using the PC membrane reached not more than $40 \%$ dissolved substance. In contrast, the dissolution profile for budesonide using the IPC membrane reached $80 \%$ dissolved. These results confirm that the effect of lower permeability is membrane dependent and not setup dependent.

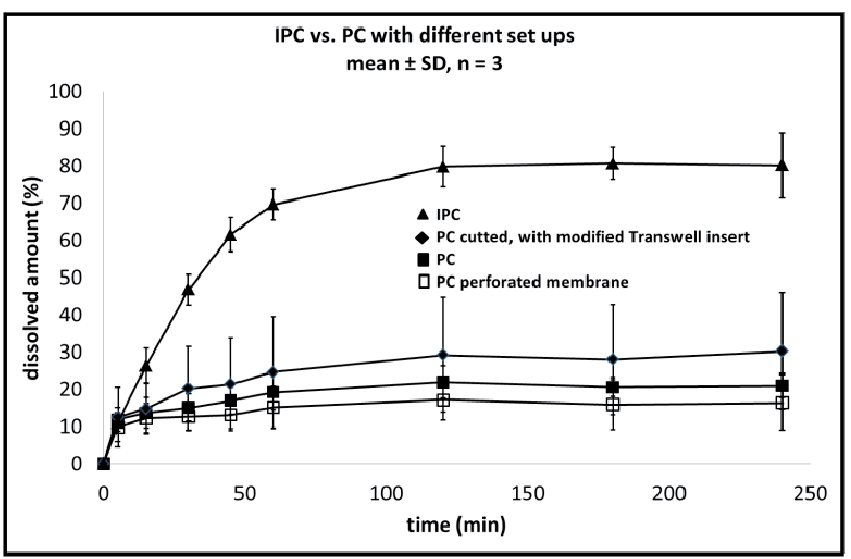

Figure 9. Budesonide dissolution profiles using the PC membrane and the IPC membrane: ( $\mathbf{\Delta})$ IPC; ( $)$ PC Transwell membrane cut and placed in modified Transwell insert; (ם) PC Transwell insert; and ( $\square$ ) PC Transwell insert perforated edge (mean $\pm S D, n=3$ ).

\section{DISCUSSION}

The investigation and determination of different crucial parameters for a miniaturized dissolution setup for inhalation powders was the focus of this study. As this is especially important for drugs of low solubility, two model drugs, budesonide and substance A, were chosen. Substance $A$ is an actual Boehringer Ingelheim research compound that is also available in different forms (crystalline base, amorphous base, and dibromide). Previously, we could demonstrate that a key parameter is the deposition of the drug on the membrane (11). This parameter is therefore addressed using an abbreviated $\mathrm{ACl}$ with stage extension between stage one and the filter stage allowing homogeneous deposition of the powder on the test membranes. Testing possible drug-membrane interactions assessing the membrane permeability of pure and dissolved drug is essential before performing dissolution tests (Figure 5). The dissolution profile will always be affected by the dissolution of the powder on the membrane and the interaction with and diffusion across the membrane. To separate the processes, a diffusion test is meaningful to address material interactions (13, 14). Permeability testing suggests that diffusion has a strong influence on the dissolution profile depending on the membrane. The large deviations do not allow a comparison of the diffusion for one drug among the different membranes. Nevertheless, such data indicate the 
total amount and speed of the overall process. Therefore, the suitability of a membrane for the drug dissolution test can be determined. The PE membrane showed the slowest permeability. A clear substance dependence and a large difference between the diffusibility of PC and IPC membranes was also seen. In addition to the type of material, the SEM images (Figure 4) demonstrate the different diffusion areas (porosities) that influence the profiles. The PE membrane pores have only $0.5 \%$ coverage of the whole membrane area. The area is $12.6 \%$ for PC and $18.9 \%$ for IPC, resulting in different permeation areas of $3.9 \times 10^{-3} \mathrm{~cm}^{2}, 0.1 \mathrm{~cm}^{2}$, and $0.15 \mathrm{~cm}^{2}$ for $P E, P C$, and $I P C$, respectively. For budesonide and substance $A$ dibromide, the permeability behavior correlates with the different permeation areas (small area $\rightarrow$ slow diffusion). However, for substance $A$ amorphous base, the diffusion velocity on the PE membrane was much larger than the diffusion across the PC membrane despite the larger diffusion area. This points to a specific interaction between the membrane and the drug, which was also reported by Bhagwat et al. (16). Due to the substance-specific differences in membrane permeation tests (Figure 5) and a larger difference in the dissolution profiles for all substances (Figure 8), a direct comparison between the two membranes (IPC and PC) for budesonide was performed (Figure 9). PC (original Transwell membrane) and IPC membranes showed comparable pore numbers per $\mathrm{cm}^{2}\left(\mathrm{PC}=1 \times 10^{8}\right.$ pores $/ \mathrm{cm}^{2} ; \mathrm{IPC}=1.5 \times 10^{8}$ pores $/$ $\mathrm{cm}^{2}$ ) and a similar appearance (Figure 4), which was obviously not responsible for the observed differences. Another difference was in the setup itself. The modified insert with a sieve was used for the IPC membrane. Consequently, there was a small gap between membrane and insert wall that is not present in the commercial inserts. To match the experimental conditions, the PC membrane was cut out after dose collection and placed into the modified insert. However, the dissolution profiles again demonstrate this large difference between PC and IPC membranes (Figure 9). Additionally, the PC membrane of the commercial setup was perforated at the edge to simulate the gap and to test the influence on the diffusion. Surprisingly, no effect was found with this setup. In this context, the membrane polarity was investigated to see if possible wetting differences could explain this behavior. The contact angle measurements with water underline the hydrophilic character of all membranes. However, the higher variability of the advancing contact angles for the PC membranes $\left(\theta_{\mathrm{PC}} \in[56-76], \theta_{\mathrm{IPC}} \in\right.$ [54-58]) indicates that there might be hydrophilic and hydrophobic hot spots as a result of surface modification by the manufacturer for tissue culturing. Such membrane surfaces become hydrophilic and negatively charged when medium is added (21). Furthermore, the surfaceadsorbed compounds might interact with the substances thereby hindering substance diffusion through the PC membrane.

Besides the membrane, contact of the powder with the dissolution medium to facilitate the dissolution process is critical. Because of the small amount of dissolution medium in and on the membrane, the impact of additional dissolution medium on the membrane and thus on the dissolution process was determined. We expected faster and less variable dissolution profiles, as described by Arora et al. $(13,14)$. However, the impact of additional dissolution medium on the membrane is substance-dependent, and in most cases leads to higher variability in the dissolution process. The hydrophilic nature of the membrane fosters the assumption that the liquid is uniformly distributed on the membrane (24-mm diameter) completely covering the inhalable particles (40 $\mu \mathrm{L}$ PBS buffer results in $88-\mu \mathrm{m}$ solvent layer). Nevertheless, the particles on the membrane still dissolve slowly, even slightly slower than without the additional layer of water. Thus, the absence of additional dissolution medium is not a crucial factor.

Stirring is supposed to have a positive effect on the reproducibility and speed of the dissolution process (16), but currently no direct comparison for the Transwell system with or without stirring has been reported. The concentration of API is highest directly below the membrane in the acceptor compartment, and without stirring the dissolution might be limited because of the high concentration below and in the membrane. Hence, diffusion of API to areas with lower concentration in the acceptor medium is the rate-determining step. With stirring, a higher and homogeneous concentration gradient through the membrane is established (21) and kept high. Thus, the rate-determining step is the dissolution of the particles. Therefore, the Transwell setup was modified by inserting a spacer to lift the inserts, allowing the addition of stir bars. Furthermore, for substance-specific comparison of dissolution profiles, it is mandatory that particle size distribution, amount of drug loading on membrane, and particle distribution on the membrane are similar $(8,11-13)$. From Table 4 it can be seen that membrane loading is very similar for PC and PE membranes. Hence, differences in the diffusion profiles are not mass effects but originate from the method. A comparison of IPC (Figure 6c) and RC (Figure 6b) for the stirred and non-stirred setups has to be done carefully, because of significant different masses on the membrane. Interestingly, a lower deposited mass on the 
RC membrane under non-stirred conditions leads to a slower dissolution process than for the IPC membrane with stirring and a larger mass. This surprising behavior might be due to unknown material interactions. For the IPC membrane, a detailed focus on the substances is necessary. For substance A crystalline base, the absence of any stirring effect is most likely based on the poor solubility (over $50 \%$ less than budesonide). This is supported by the fact that the mass was adjusted to equal amounts and the mass distribution was controlled to be homogenous on the membrane thus not influencing the dissolution profile. For budesonide the large difference between the dissolution profiles could be explained by the positive effect of stirring, as the particle mass on the membrane is similar and hence has no effect. In summary, for most substances tested, stirring will lead to better reproducibility and faster dissolution profiles as expected.

Looking at the MDT as a possible determinant for the dissolution process Table 4 reveals that for most setups, the MDT reflects the dissolution profile rank order. This allows good discrimination among different dissolution profiles obtained under different experimental settings. Nevertheless, in several cases MDT and dissolution profiles are contradictory, for example, budesonide and substance A dibromide (RC, without additional dissolution medium on membrane). This behavior is most likely due to the different phases of the dissolution process not reaching a plateau and hence hindering the determination of the correct MDT value.

For budesonide, the data clearly demonstrate the impact of the different parameters tested and the need for carefully pretesting the possible determinants of the dissolution setup. Without such a pre-evaluation, the dissolution profiles might lead to misleading conclusions by not eliminating or at least considering setupdependent effects as most likely happened previously for the dissolution of budesonide (13).

\section{CONCLUSION}

The results of this study show that the membrane material, the amount of dissolution medium on the membrane, and stirring of the acceptor medium are critical factors for dissolution testing of pharmaceutical aerosols particles after deposition on a Transwell system. To avoid false conclusions and misleading data, it is of utmost importance to investigate the specific interactions of the drug used with the dissolution setup materials.

The results show that the membrane material strongly influences the dissolution process. Additionally, the PE membrane has a substance-retaining effect. Furthermore, additional dissolution medium on the membrane is not suitable for the poorly soluble drugs used because of a reduction in the reproducibility of the dissolution process. Stirring of the dissolution medium is beneficial; it leads to a faster dissolution with better reproducibility. Moreover, an optimal discriminating and reproducible setup (i.e., IPC membrane, stirring, no addition of dissolution medium on the membrane) for the four tested substances was identified.

\section{CONFLICT OF INTEREST}

No conflict of interest has been declared by the authors.

\section{REFERENCES}

1. Labouta, H. I.; Schneider, M. Tailor-made biofunctionalized nanoparticles using layer-by-layer technology. Int. J. Pharm. 2010, 395 (1-2), 236-242. DOI: 10.1016/j.ijpharm.2010.05.019.

2. <601> Aerosols, Nasal Sprays, Metered-Dose Inhalers, and Dry Powder Inhalers. In The United States Pharmacopeia and National Formulary USP 36-NF 31; The United States Pharmacopeial Convention, Inc.: Rockville, MD, 2013.

3. European Pharmacopoeia, 7th ed., Supplement 7.7; European Directorate for the Quality of Medicines \& Healthcare, Council of Europe: Strasbourg, France, 2013.

4. Gray, V. A.; Hickey, A. J.; Balmer, P.; Davies, N. M.; Dunbar, C.; Foster, T. S.; Olsson, B. L.; Sakagami, M.; Shah, V. P.; Smurthwaite, M. J.; Veranth, J. M.; Zaidi, K. The Inhalation Ad Hoc Advisory Panel for the USP performance tests of inhalation dosage forms. Pharm. Forum 2008, 34 (4), 1068-1074.

5. Henning, A.; Hein, S.; Schneider, M.; Bur, M.; Lehr, C.M. Pulmonary Drug Delivery: Medicines for Inhalation. In Handbook of Experimental Pharmacology; SchäferKorting, M., Ed.; Springer-Verlag: Heidelberg, 2010; Vol. 197, p 171.

6. Jensen, B.; Reiners, M.; Wolkenhauer, M.; Ritzheim, P.; May, S.; Schneider, M.; Lehr, C.-M. In Dissolution Testing For Inhaled Products; Dalby, R., Byron, P., Peart, J., Suman, J., Young, P., Eds.; DHI Publishing: Berlin, 2011; pp 303-308.

7. Davies, N. M.; Feddah, M. R. A novel method for assessing dissolution of aerosol inhaler products. Int. J. Pharm. 2003, 255 (1-2), 175-187. DOI:10.1016/S03785173(03)00091-7.

8. May, S.; Jensen, B.; Wolkenhauer, M.; Schneider, M.; Lehr, C. M. Dissolution Techniques for In Vitro Testing of Dry Powders for Inhalation. Pharm. Res. 2012, 29 (8), 2157-2166. DOI:10.1007/s11095-012-0744-2.

9. Salama, R. O.; Traini, D.; Chan, H.-K.; Young, P. M. Preparation and characterisation of controlled release co-spray dried drug-polymer microparticles for inhalation 2: Evaluation of in vitro release profiling methodologies for controlled release respiratory 
aerosols. Eur. J. Pharm. Biopharm. 2008, 70 (1), 145152. DOI:10.1016/j.ejpb.2008.04.009.

10. Haghi, M.; Traini, D.; Bebawy, M.; Young, P. M. Deposition, Diffusion and Transport Mechanism of Dry Powder Microparticulate Salbutamol, at the Respiratory Epithelia. Mol. Pharmaceutics 2012, 9 (6), 1717-1726. DOI:10.1021/mp200620m.

11. May, S.; Jensen, B.; Weiler, C.; Wolkenhauer, M.; Schneider, M.; Lehr, C.-M. Dissolution Testing of Powders for Inhalation: Influence of Particle Deposition and Modeling of Dissolution Profiles. Pharm. Res. 2014, 31 (11), 3211-3224. DOI:10.1007/s11095-014-1413-4.

12. Son, Y.-J.; Horng, M.; Copley, M.; McConville, J. T. Optimization of an In Vitro Dissolution Test Method for Inhalation Formulations. Dissolution Technol. 2010, 17 (2), 6-13. DOI: 10.14227/DT170210P6.

13. Arora, D.; Shah, K. A.; Halquist, M. S.; Sakagami, M. In Vitro Aqueous Fluid-Capacity-Limited Dissolution Testing of Respirable Aerosol Drug Particles Generated from Inhaler Products. Pharm. Res. 2010, 27 (5), 786795. DOI 10.1007/s11095-010-0070-5.

14. Sakagami, M.; Arora Lakhani, D. Understanding Dissolution in the Presence of Competing Cellular Uptake and Absorption in the Airways. Proceedings of Respiratory Drug Delivery 2012, Phoenix, AZ, May 1317, 2012; Dalby, R. N., Byron, P. R., Peart, J., Suman, J.D., Farr, S.J., Young, P.M., Eds.; Respiratory Drug Delivery: Richmond, VA, 2012; pp 185-192.

15. Haghi, M.; Traini, D.; Young, P. In Vitro Cell Integrated Impactor Deposition Methodology for the Study
Of Aerodynamically Relevant Size Fractions from Commercial Pressurised Metered Dose Inhalers. Pharm. Res. 2014, 31 (7), 1779-1787. DOI:10.1007/s11095-0131282-2.

16. Bhagwat, S.; Rohrschneider, M.; Alfadehl, S.; Hochhaus, G. Development of An in Vitro Test Method for Dissolution of Inhaled Corticosteroids. J. Aerosol Med. Pulm. Drug Delivery 2013, 26 (2), A58-A59. DOI: 10.1089/jamp.2013.00A1.

17. Franz, T. J. PERCUTANEOUS ABSORPTION. ON THE RELEVANCE OF IN VITRO DATA. J. Invest. Dermatol. 1975, 64 (3), 190-195. DOI: 10.1111/1523-1747.ep12533356.

18. Langguth, D.; Fricker, G.; Wunderli-Allenspach, $\mathrm{H}$. Biopharmazie; Wiley-VCH Verlag $\mathrm{GmbH}$ : Weinheim, Germany, 2004.

19. Dissolution Testing of Immediate Release Solid Oral Dosage Forms; Guidance for Industry; U.S. Department of Health and Human Services, Food and Drug Administration, Center for Drug Evaluation and Research (CDER), U.S. Government Printing Office: Washington, DC, 1997.

20. Guideline on the Investigation of Bioequivalence; CPMP/ EWP/QWP/1401/98 Rev. 1; Committee for Medicinal Products for Human Use (CHMP), European Medicines Agency: London, 2010.

21. Ryan, J. A. Evolution of Cell Culture Surfaces. BioFiles 2008, 3.8 (21), 1-5. http://www.sigmaaldrich.com/ technical-documents/articles/biofiles/evolution-of-cell. html (accessed July 21, 2015). 